\title{
The effects of extreme conditions on molecular solids
}

Iain D. H. Oswald ${ }^{a}$ and Christine M. Beavers $\underline{\underline{b}}$

aStrathclyde Institute of Pharmacy and Biomedical Sciences, University of Strathclyde, 161 Cathedral Street, Glasgow, UK. E-mail: iain.oswald@strath.ac.uk

bDiamond Light Source Ltd, Diamond House, Harwell Science \& Innovation Campus, Didcot, Oxfordshire, OX11 ODE, UK. E-mail: christine.beavers@diamond.ac.uk

The effect of high pressure on molecular systems has been extensively studied in the last twenty years. Over this period there has been an expansion of the types of materials under study from simple alcohols $\mathrm{s}^{1,2}$ and amino acids ${ }^{3-5}$ towards more complex systems ${ }^{6,7}$. In most of these studies, pressure has been able to induce transitions in materials that have not been observed via other ambient pressure techniques: simple molecular systems demonstrate changes in hydrogen-bonding, rotational configurations, and even display new chemical bonds.

This themed issue includes 12 articles that expand over a wide range of materials and pressures. Throughout this issue, the methods employed by the authors are demonstrative of those used to characterise materials under extreme conditions; the boundaries are being pushed to extremes, whether it is the size and complexity of the molecules, the techniques themselves or the pressures achieved.

High-pressure techniques to investigate the polymorphism of low melting compounds were employed by Smith et al (DOI: 10.1039/C9CE00870E) and Allan and Barnett (DOI:

$10.1039 /$ C9CE00485H). Smith et al. explored the changes in the packing behaviour of anisole and discovered that the packing efficiency in the high-pressure form outweighed any electrostatic interaction in the structures. Allan and Barnett explored the crystallisation of trifluoroethanol building on their extensive work on alcohols whilst tapping into the growing field of halogen-bonding with the introduction of the trifluoro- group. Interestingly trifluoroethanol mimics the packing of the high pressure pahse of ethanol. In both these cases the packing of the molecules was promoted at the expense of more elongated electrostatic or hydrogen bonding interactions which is commensurate with other studies of similar system e.g. phenol. ${ }^{8}$

Following on from these studies there are three studies investigating biomimetic materials; glycine, threonine and sodium salt of guanine. Hinton et al (DOI: 10.1039/C8CE02123F) investigated the pressure dependence of alpha-glycine to a pressure of $50 \mathrm{GPa}$ using a combination of X-ray powder diffraction and DFT calculations. Through their study they were able to note the stability of alphaglycine to this maximum pressure and really pushing the boundaries of the study of organic materials to ever higher pressures. The extremes of pressure continue with the study by Giordano and co-workers on threonine (DOI: 10.1039/ C9CE00388F). Through a combination of single crystal X-ray, neutron powder diffraction and DFT calculations the authors were able to determine three phase transitions to $22 \mathrm{GPa}$. Using Raman studies as a guide, ${ }^{9}$ the authors were able to provide structural information and demonstrate that two of the transitions were isosymmetric and involved subtle reorientation of functional groups or a change in which those groups interacted in the crystal structure. One of the key outcomes was that even at these extremes of pressure the hydrogen bonding remained within the bounds set by ambient pressure crystal structures. Finally, for the biomimtic materials, Gaydamaka and coworkers (DOI: 10.1039/ C9CE00476A) explored the pressure dependence of a disodium guanine heptahydrate salt (2Na+· $55 \mathrm{H} 3 \mathrm{~N} 5 \mathrm{O} 2-\cdot 7 \mathrm{H} 2 \mathrm{O})$. In this study the team observed that this salt form undergoes a phase transformation at $2.5 \mathrm{GPa}$ that is reconstructive in nature. The authors took care to explore the avenues of the selection of pressure 
transmitting medium to ensure the transition was material related and not due to the medium. They demonstrated that the phase transformation was partially reversible.

Pushing the bounds of complexity, Funnell et al explored the orange plate polymorph of 5-Methyl-2[(2-nitrophenyl)-amino]-3-thiophenecarbonitrile, or ROY as it is more commonly referred (DOI: 10.1039/C8CE01946K). Due to the complexity in the molecule, deuteration was not possible hence the study was performed on a hydrogenated material using neutron powder diffraction which is pushing the capabilities of the technique to an extreme. This is also a theme in the study of Liu et al who were investigating the polymorphism in methyl stearate and observed four phase transformations to $6.3 \mathrm{GPa}$ (DOI: 10.1039/C9CE00393B). They were able to observe transitions using $X$-ray powder diffraction but were limited in the neutron powder study due to the narrower window of d-spacing and very large cell (95 Å cell axis!). Connor et al explored the co-crystal of indomethacin and saccharin as a tool to probe different hydrogen bonding motifs not easily observed in single component systems (DOI: 10.1039/C9CE00838A). The host-guest nature of the structure promoted different behaviour of the hydrogen bonding. Fanetti et al. explored the chemical reaction of 2,4,6tricyano-1,3,5-triazine compression under high pressure and temperature conditions (DOI: 10.1039/C8CE02154F). Using both hydrostatic and non-hydrostatic pressure they were able to observe $45 \%$ completion to the proposed reaction product based on a triazine graphitic carbon nitrides.

New strategies were employed by Ward and Oswald to aid the recovery of high-pressure forms of paracetamol through antisolvent addition at high pressure (DOI: 10.1039/C9CE00365G). Through this they demonstrated the preservation of the orthorhombic paracetamol from a high-pressure crystallisation. During their study they also noted the appearance of a new polymorph of a methanol solvate. Different techniques were employed to investigate the behaviours of tetracene. Wang and co-workers used impedance spectroscopy to investigate the role of grain boundaries on the dielectric properties to $30 \mathrm{GPa}$ (DOI: 10.1039/ C9CE00961B). They observed that resistivity changes associated with band gap whilst the grain boundary was affected by defects. Novel materials of $\mathrm{MoS}_{2} \mathrm{ReS}_{2}$ were prepared by Anenburg at $1 \mathrm{GPa}$ and $800^{\circ} \mathrm{C}$ through the use of carbonate melts and characterised by Scanning electron microscopy and wavelength-dispersive X-ray Spectroscopy (DOI: 10.1039/C9CE00188C).

We extend our thanks to the authors who contributed to this special issue and also to the CrystEngComm team for all their help with respect to the issue. R11809-R11812. Crystallogr. Sect. B-Structural Sci., 2010, 66, 458-471. 
7 E. Eikeland, M. K. Thomsen, S. R. Madsen, J. Overgaard, M. A. Spackman and B. B. Iversen, Chem. - A Eur. J., 2016, 22, 4061-4069.

8 D. R. Allan, S. J. Clark, A. Dawson, P. A. McGregor and S. Parsons, Acta Crystallogr. Sect. B, 2002, 58, 1018-1024.

9 R. O. Holanda, J. A. Lima, P. T. C. Freire, F. E. A. Melo, J. Mendes Filho and A. Polian, J. Mol. Struct., 2015, 1092, 160-165. 